\title{
612.
}

\section{NOTE SUR UNE FORMULE D'INTÉGRATION INDÉFINIE.}

[From the Comptes Rendus de l'Académie des Sciences de Paris, tom. LxxviII. (JanvierJuin, 1874), pp. 1624-1629.]

EN étudiant les Mémoires de M. Serret (Journal de Liouville, t. x., 1845) par rapport à la représentation géométrique des fonctions elliptiques, avec les remarques de M. Liouville sur ce sujet, je suis parvenu à une formule d'intégration indéfinie qui me paraît assez remarquable, savoir: en prenant $\theta$ entier positif quelconque, je dis que l'intégrale

a une valeur algébrique

$$
\int \frac{(x+p)^{m+n-\theta}(x+q)^{\theta} d x}{x^{m+1}(x+p+q)^{n+1}}
$$

$$
(x+p)^{m+n-\theta+1}(x+p+q)^{-n} x^{-m}\left(A+B c+C x^{2}+\ldots+K x^{\theta-1}\right),
$$

pourvu qu'une seule condition soit satisfaite par les quantités $m, n, p, q$. Cette condition s'écrit sous la forme symbolique

en dénotant ainsi l'équation

$$
\left([m] p^{2}+[n] q^{2}\right)^{\theta}=0,
$$

$$
[m]^{\theta} p^{2 \theta}+\frac{\theta}{1}[m]^{\theta-1}[n]^{1} p^{2 \theta-2} q^{2}+\ldots+[n]^{\theta} q^{2 \theta}=0,
$$

où, comme à l'ordinaire, $[m]^{\theta}$ signifie $m(m-1) \ldots(m-\theta+1)$.

Je rappelle que les formules de M. Serret ne contiennent que des exposants entiers, et celles de M. Liouville qu'un seul exposant quelconque: la nouvelle formule contient deux exposants quelconques, $m, n$. Je remarque aussi l'analogie de la condition $\left([m] p^{2}+[n] q^{2}\right)^{\theta}=0$ avec celle-ci

$$
\frac{1}{\zeta^{n-m}}\left(\frac{d}{d \zeta}\right)^{m} \zeta^{n}(\zeta-1)^{m}=0
$$

( $m$ étant un entier positif), qui figure dans les Mémoires cités. 
Pour démontrer la formule, j'écris

$$
u=x^{-m}\left(A+B x+C x^{2}+\ldots+K x^{\theta-1}\right),
$$

et aussi pour abréger

$$
X=(x+p)^{m+n-\theta+1}(x+p+q)^{-n}
$$

ce qui donne

$$
\frac{X}{(x+p)(x+p+q)}=(x+p)^{m+n-\theta}(x+p+q)^{-n-1} .
$$

L'équation à vérifier est donc

$$
X u=\int \frac{X(x+q)^{\theta} d x}{x^{m+1}(x+p)(x+p+q)},
$$

ou, en différentiant et divisant par $X$,

$$
\frac{X^{\prime}}{X} u+u^{\prime}=\frac{(x+q)^{\theta}}{x^{m+1}(x+p)(x+p+q)},
$$

ou enfin

$$
[(m+n-\theta+1)(x+p+q)-n(x+p)] u+(x+p)(x+p+q) u^{\prime}=\frac{(x+q)^{\theta}}{x^{m+1}},
$$

où $u^{\prime}$ dénote $\frac{d u}{d x}$. Il ne s'agit donc que d'exprimer que cette équation ait une intégrale

$$
u=x^{-m}\left(A+B x+C x^{2}+\ldots+K x^{\theta-1}\right) .
$$

En supposant que cela soit ainsi, et en effectuant la substitution, les termes en $x^{-m+\theta}$ se détruisent, et l'on obtient une équation qui contient des termes en $x^{-m-1}$, $x^{-m}, \ldots, x^{-m+\theta-1}$, savoir $(\theta+1)$ termes. On a ainsi, entre les $\theta$ coefficients $A, B, C, \ldots$, $K$ un système de $(\theta+1)$ équations linéaires, ce qui implique une condition entre les constantes $m, n, p, q$; mais, cette condition satisfaite, les équations se réduisent à $\theta$ équations indépendantes, et les coefficients seront ainsi déterminés.

Par exemple, soit $\theta=2$; l'équation différentielle est

$$
[\overline{m-1} p+\overline{m+n-1} q+\overline{m-1} x] u+\left[p^{2}+p q+x(2 p+q)+x^{2}\right] u^{\prime}=x^{-m-1}(q+x)^{2},
$$

laquelle doit être satisfaite par $u=A x^{-m}+B x^{-m+1}$. Cela donne

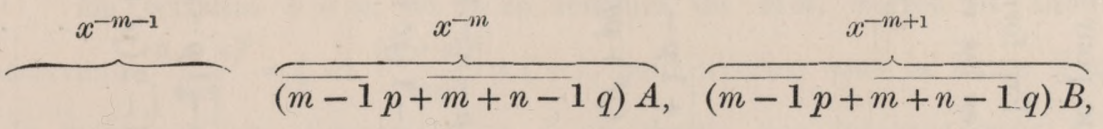

$$
\begin{aligned}
& (m-1) A, \quad(m-1) B \\
& -m\left(p^{2}+p q\right) A, \quad-(m-1)\left(p^{2}+p q\right) B, \\
& -m(2 p+q) A, \quad-(m-1)(2 p+q) B, \quad " \quad=0, \\
& -q^{2} \quad, \quad-2 q \\
& -m A, \quad-(m-1) B \\
& -1 \text {, },
\end{aligned}
$$



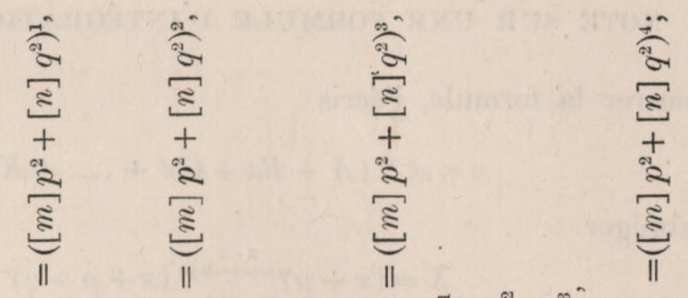

$\stackrel{\Xi}{\sharp} \quad \vdots$

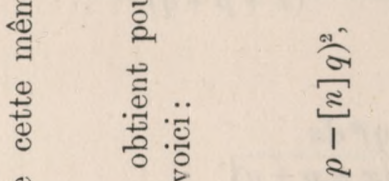

$\stackrel{0}{0}$

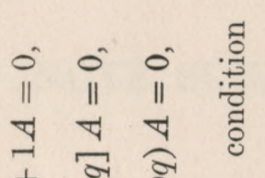

ซี

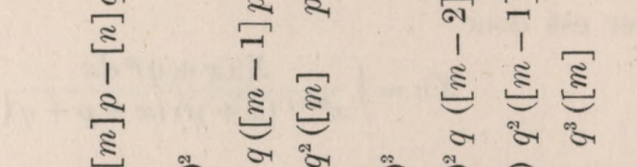

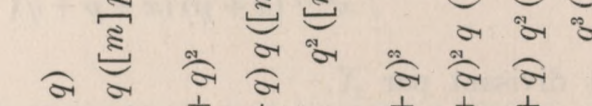

$$
\begin{aligned}
& 2 \\
& + \\
& 3 \\
& 2
\end{aligned}
$$

$+2+$

33

$+++$

3 33

है है 2

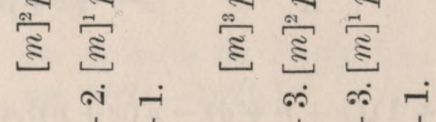

II +1

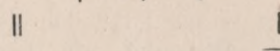

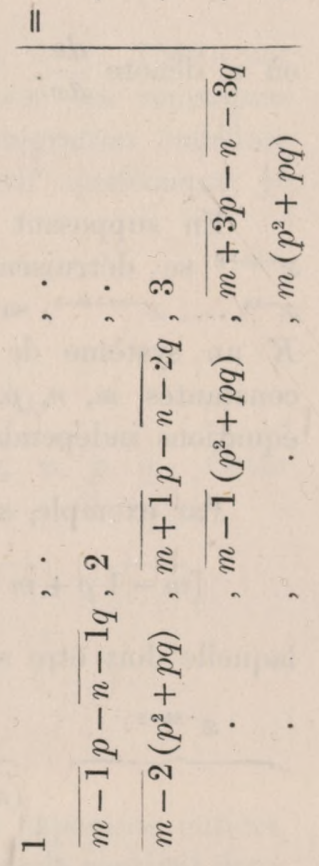

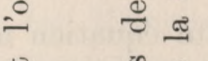

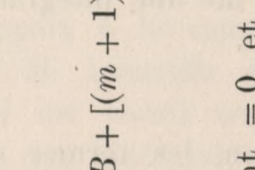

० $\quad$ वे एँ

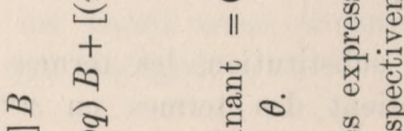

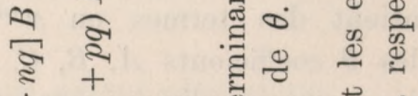

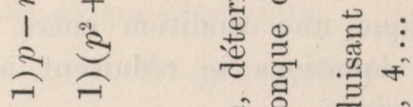

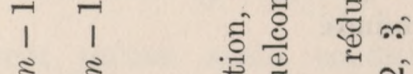

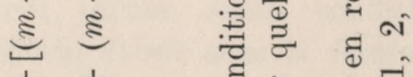

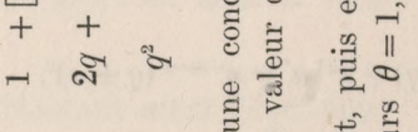

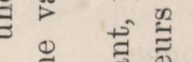

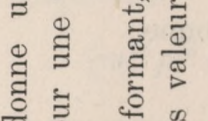

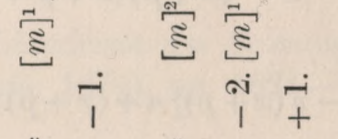

॥

I

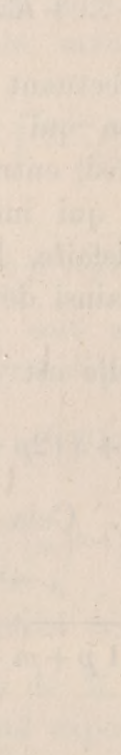

or है है है.

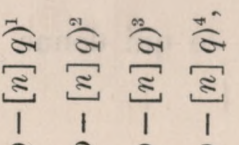
(4) 25

के ने ने के

$++++$

3 \& 33

है, है, है, 2

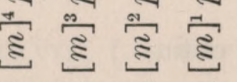

$\begin{array}{rrr}1+1 & + \\ 1+ & +\end{array}$

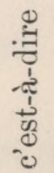

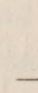

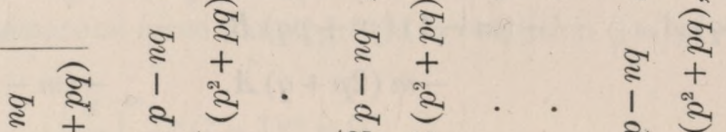

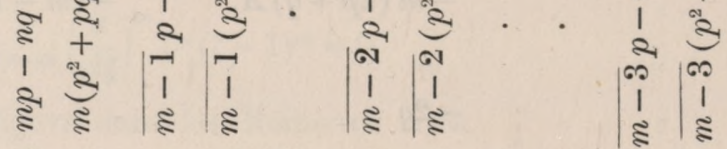

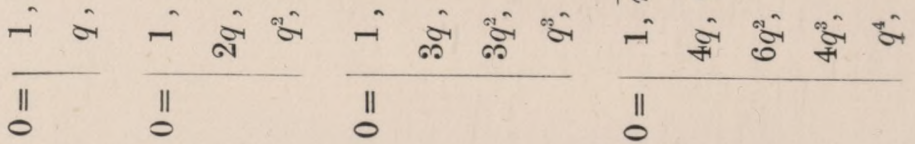


et ainsi de suite. Les notations $([m] p-[n] q)^{1},([m] p-[n] q)^{2}, \ldots$ ont des significations semblables à celles de $\left([m] p^{2}+[n] q^{2}\right)^{1},\left([m] p^{2}+[n] q^{2}\right)^{2}, \ldots$, auparavant expliquées. On a, par exemple,

$$
([m] p-[n] q)^{2}=[m]^{2} p^{2}-2[m]^{1}[n]^{1} p q+[n]^{2} q^{2} .
$$

Considérons, par exemple, le deuxième déterminant: ceci contient trois termes en $1,2 q, q^{2}$ respectivement; le premier terme est

c'est-à-dire

$$
\text { 1. }(m-1)\left(p^{2}+p q\right) \cdot m\left(p^{2}+p q\right)
$$

le deuxième terme est

$$
[m]^{2} p^{2}(p+q)^{2} ;
$$

c'est-à-dire

$$
2 q \cdot-m\left(p^{2}+p q\right)[(m-1) p-n q]
$$

le troisième terme est

$$
-2[m]^{1} p(p+q) q([m-1] p-[n] q)^{1} ;
$$

c'est-à-dire

$$
q^{2}\left[(\overline{m-1} p-n q)(\overline{m+1} p-\overline{n-1} q)-(m-1)\left(p^{2}+p q\right)\right]
$$

$$
q^{2}\left[\left(m^{2}-m\right) p^{2}-2 m n p q+\left(n^{2}-n\right) q^{2}\right]=q^{2}([m] p-[n] q)^{2} .
$$

Et de même le troisième déterminant est composé de quatre termes en $1,3 q, 3 q^{2}, q^{3}$ respectivement, lesquels sont les quatre termes de la première expression transformée; et ainsi pour le quatrième déterminant, etc. Au moyen de ces premières transformées, on obtient sans peine les expressions finales $\left([m] p^{2}+[n] q^{2}\right)^{1},\left([m] p^{2}+[n] q^{2}\right)^{2}, \ldots$ devient

En écrivant $z-\frac{1}{2}(p+q)$ au lieu de $x$, et puis $\frac{1}{2}(p+q)=\alpha, \frac{1}{2}(p-q)=a$, la formule

et la valeur algébrique

$$
\int \frac{(z-a)^{\theta}(z+a)^{m+n-\theta} d \theta}{(z-\alpha)^{m+1}(z+\alpha)^{n+1}}
$$

$$
=(z+a)^{m+n-\theta-1}(z-\alpha)^{-m}(z+\alpha)^{-n}\left(A^{\prime}+B^{\prime} z+\ldots+K^{\prime} z^{\theta-1}\right),
$$

pourvu qu'on ait entre les quantités $m, n, a, a$ la relation

$$
\left\{[m](\alpha+a)^{2}+[n](\alpha-a)^{2}\right\}=0 .
$$

En écrivant $\theta=m$, on a la formule de MM. Serret et Liouville, laquelle, en y écrivant $\frac{(\alpha+a)^{2}}{4 a \alpha}=\zeta$ et $\frac{(\alpha-a)^{2}}{4 a \alpha}=\zeta-1$, peut s'écrire sous la forme $\{[m] \zeta+[n](\zeta-1)\}^{\theta}=0$. Je remarque que l'équation en $\zeta$ ne donne pas toujours pour $\zeta$ des valeurs réelles, positives et plus grandes que l'unité: par exemple, pour $\theta=1$, on a $\zeta=\frac{n}{m+n}$, valeur qui ne peut pas satisfaire à ces conditions. Je n'ai pas cherché dans quel cas ces conditions (qui ont rapport à l'application des formules à la représentation des fonctions elliptiques) subsistent. 